EUTOMIA

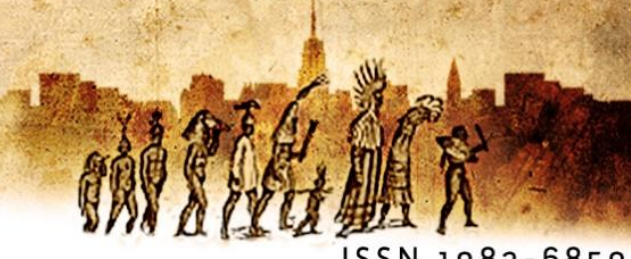

ISSN $1982-6850$

\title{
El dialogismo y los géneros de la interacción verbal humana en Jakubinskij y Voloshinov
}

\author{
Dora Riestra' (Universidad Nacional de Río Negro)
}

Resumo: Voloshinov e Jakubinskij, dois autores russos referenciais do campo de estudo da interação humana, cujas contribuições foram reconhecidas neste século, a partir de pesquisas históricas e epistemológicas de linguistas eslavistas (em particular, Ivanova e Sériot), são revisados em função de elaborar propostas educacionais no ensino das linguas. $O$ contexto cultural russo foi levado em consideração, tanto na precisão terminológica, quanto no reconhecimento de tradições e influências particulares. Colocam-se algumas questões de tradução e edição de dois trabalhos dos autores que contribuem conceitualmente para os estudos do diálogo em sala de aula e para questões relacionadas aos gêneros textuais como objetos de ensino. $O$ interesse em aprofundar 0 estudo da formação e internalização da linguagem externa, conceito central da atividade da linguagem, continua sendo nosso objeto de pesquisa didática. As metodologias descritas apontam para a discussão dos problemas atuais de interação e ensino das línguas.

Palavras-chave: interação humana; diálogo; gêneros; atividade da linguagem; ensino de línguas

Resumen: Voloshinov y Jakubinskij, dos autores rusos referenciales del campo de estudio de la interacción humana, cuyas contribuciones fueran reconocidas en este siglo, a partir de investigaciones históricas y epistemológicas de lingüistas eslavistas (en particular, Ivanova y Sériot), serán revisados en función de elaborar propuestas educacionales en la enseñanza de las lenguas. El contexto cultural ruso será considerado tanto en la precisión terminológica como en el reconocimiento de tradiciones y metodologías particulares. Se colocan algunas cuestiones de traducción y de edición de dos trabajos de los autores que contribuyen conceptualmente para los estudios del diálogo en el aula y sobre los géneros textuales como objetos de enseñanza. El interés en profundizar el estudio de la formación y la internalización del lenguaje externo, concepto central de la actividad de lenguaje, continúa siendo el centro de nuestro 
objeto de investigación didáctica. Las metodologías descriptas apuntan a discutir los problemas actuales de interacción y enseñanza de las lenguas.

Palabras clave: interacción humana; diálogo; géneros; actividad del lenguaje; enseñanza de lenguas.

\section{Introducción contextual}

El origen de los conceptos y su desarrollo en la historia cultural humana no son procesos lineales, por el contrario, podemos decir que se trata de caminos sinuosos, circunstanciales y, muchas veces, casuales, por oportunidad gnoseológica o, a veces, por cuestiones tan prosaicas como las políticas editoriales.

A partir de la década del 90 del siglo pasado en investigaciones educativas occidentales, los autores referenciales del ámbito educacional en el campo de estudios de la interacción humana fueron Vygotski con sus discípulos Luria y Leontiev, aunque algunas traducciones de sus obras más conocidas comenzaron a circular desde los años setenta en los países latinoamericanos, por lo general en ediciones de divulgación muy restringida, debido a cuestiones de índole política. Dicho de otro modo, los libros de la URSS no entraban legalmente. En lo que respecta a Voloshinov y Jakubinskij, sus referencias educacionales son más recientes y sus aportes han ido reconociéndose lentamente desde que comenzó la divulgación de autores rusos mediante traducciones realizadas por lingüistas que realizaron investigaciones históricas y epistemológicas de la década 20-30 del siglo pasado.

En el caso de Voloshinov (1985-1936), hubo una suerte de negación de autoría. Su obra Marxismo y filosofía del lenguaje, cuya publicación en la URSS data de 1929, le fue injustamente atribuida a Bajtín o a un círculo de discípulos suyos (situación inexistente, hoy finalmente corroborada con datos históricos), quienes habrían sido autores, colectivamente, de la obra de Voloshinov. Este malentendido produjo un relato mítico, debido a que a fines de los años 60 en Francia se atribuía un sentido ideológico grupal a la producción de autores soviéticos apenas conocidos.

De este modo, la autoría de Voloshinov -quien muriera tempranamente de tuberculosis- quedó eclipsada en occidente por el fenómeno divulgativo de Bajtin que hiciera Julia Kristeva durante el mayo francés de 1968 (SÉRIOT, 2010; BUBNOVA, 2009; TYLKOWSKI, 2012). 
Por su parte, Jakubinskij, recién en 2003 fue conocido en Europa central a través de la divulgación de Sériot e Ivanova, con la traducción al francés en 2012 de Lev Jakubinskij, une linguistique de la parole, que fue traducido al portugués en 2015 como Sobre a fala dialogal.

No obstante su reciente divulgación entre nosotros, la lectura de estos autores rusos de la década 20-30 de la URSS nos abre un panorama conceptual novedoso, que nos permite acceder a concepciones e investigaciones sobre el lenguaje humano que se anticiparon a estudios disciplinares norteamericanos sobre la conversación y turnos de habla, realizados en la segunda mitad del siglo XX.

Por las investigaciones de los lingüistas eslavistas mencionados sabemos que Jakubinskij y Voloshinov, autores que nos ocupan en particular, conocían los estudios y desarrollos de investigaciones alcanzados en el área de lo que hoy llamamos ciencias humanas y sociales, tanto de Europa central como de Estados Unidos de Norteamérica.

La acumulación del conocimiento entre los intelectuales pre y posrevolucionarios de las dos primeras décadas del siglo $\mathrm{XX}$ produjo síntesis teóricas muy elaboradas, que permanecieron desconocidas por las cuestiones geopolíticas que nos afectaron durante más de medio siglo. Lo que sorprende es la asimetría del conocimiento, puesto que los rusos conocían las producciones teóricas occidentales y no a la inversa. Una hipótesis posible es la del posicionamiento eurocentrista como obstáculo político-ideológico que impidió el conocimiento de los autores comunistas, así como las tradiciones culturales e intelectuales desarrolladas en Europa del este.

Desde esta reconsideración realizada por Sériot (2008) respecto de la alteridad rusa como diferente de la China o del Japón, pero también de la Europa occidental, es que abordamos los conceptos de los rusos en sus traducciones más precisas y cuidadas para transmitir el sentido de sus producciones teóricas. Nos mueve el interés por comprender y poder utilizar la teoría en la investigación y la instrumentación conceptual en las ciencias del lenguaje para buscar soluciones a los problemas actuales de enseñanza de las lenguas. Desde esta perspectiva, en este artículo, se busca articular las concepciones y los aportes epistemológicos de los dos autores: Jakubinskij y Voloshinov, sobre el dialogismo y los géneros de la interacción verbal humana. 
Según Ivanova (2003), en la Rusia de los años veinte, la pragmática lingüística y la poética tuvieron el mismo origen. Después de la revolución del 1917, un nuevo paradigma científico con un enfoque sociológico vinculado a la metodología marxista llevó a concebir la lingüística como nueva lingüística pragmática:

bajo los tres aspectos siguientes, relacionados entre sí: como una ciencia natural - histórica - en sus relaciones con la fisiología y la acústica; como una ciencia psicológica - en sus estudios de la actividad del sujeto hablante; como una ciencia social - en sus estudios sobre la función de la lengua en la sociedad (IVANOVA, 2003).

En ese contexto Jakubinskij (2015) se refiere al diálogo como habla dialogal, natural, diferente del habla poética, artificial. Asimismo define a la escritura como artificial y monologal. Este fue el punto de partida para el análisis del diálogo como forma de la comunicación. Por abordar el aspecto del habla dialogal es que Ivanova (2003) sitúa a este autor como iniciador del campo de estudios de la comunicación.

El análisis de la forma del diálogo que Jakubinskij define en su carácter de forma fisiológica y sociológica, como lo destacara Bertau (2008), es un análisis de la forma vivida, concepto que proviene de la cultura rusa, que no es el de la forma de Jakobson, se trata de la descripción del diálogo como "fenómeno y como forma no mediatizada", un "fenómeno bio-psicológico", según expresa el mismo Jakubinskij (2015).

Por otra parte, con la finalidad de distinguir y delimitar los conceptos, tomamos la observación de Ivanova respecto del objeto estudio de Voloshinov: el dialogismo, cuya teoría aporta a los estudios del diálogo pero, además, a la estilística y al nacimiento de la lingüística del texto.

Para la lingüista rusa, el desconocimiento de ambos autores se debió al destino trágico que los marcó: la muerte de Voloshinov y el aislamiento de Jakubinskij con la interdicción de publicación de sus obras. Es necesario destacar, que Jakubinskij influyó en Voloshinov por haber sido su profesor en los estudios doctorales.

En lo que respecta a Voloshinov, se basó en Jakubinskij citándolo expresamente en Marxismo y Filosofía del lenguaje, presentándolo como el autor de la primera investigación del diálogo en Rusia y, además, tomó directamente de Jakubinskij el término "réplica interna". 
Con la lectura de los dos autores encontramos congruencia conceptual y continuidad epistemológica frente al concepto de diálogo: la forma natural de la interacción humana que conlleva una determinación extraverbal; por otra parte, encontramos la diferenciación del objeto de investigación de ambos autores: si bien Jakubinskij aborda el diálogo como fenómeno humano específico, observamos que Volsohinov avanza e incorpora el estudio del signo ideológico, el enunciado y el género, tres conceptos articulados epistemológicamente, que constituyen un aporte novedoso.

Sin el desarrollo conceptual sobre el habla dialogal y habla monologal de Jakubinskij, Voloshinov no hubiera podido construir la noción de género a partir de la entonación y sin el concepto de masas aperceptivas de Jakubinskij, la anticipación de la réplica de cada enunciado, las palabras que se utilizan y repiten (los estereotipos ) en determinadas situaciones de la vida cotidiana y que son estables relativamente, es decir, sin todos los aspectos delimitados en el texto de Jakubinskij, los "géneros verbales de la vida cotidiana" de Voloshinov no habrían surgido como concepto que aún hoy nos interpela para investigar la interacción humana.

En realidad, el conocimiento reciente de los hechos históricos en la lingüística de la Rusia soviética de los años 20-30, a través de los estudios de eslavistas como Sériot (2010, 2011, 2015), AgeevaTylkowski (2009, 2012, 2015), Ivanova (2008, 2010, 2015), Uhlik,(2011), etc., derrumbó el mito en torno a la producción colectiva, por lo tanto los textos divulgados en occidente fragmentariamente, con traducciones discutibles deberían revisarse actualmente, en función de las derivaciones teóricometodológicas.

El texto divulgado de Bajtín en español sobre los "géneros discursivos", dentro de un volumen conocido como Estética de la creación verbal, por el que se atribuyera el concepto de diálogo al mencionado autor ruso, es, en realidad, de autoría de Voloshinov.

En segundo lugar, otros autores, como es el caso de Medvedev, quien fuera fusilado en 1938, desde las investigaciones realizadas últimamente, se lo reconoce como autor de obras que le habían sido atribuidas al mito del círculo Bajtín. 
Los análisis de Bronckart y Bota (2009; 2011), en particular, Bakthine démasqué, apuntan a mostrar los hechos y a desmitificar la historia del Bajtin que aún circula en ámbitos académicos occidentales.

Seriot (2008/2010), por su parte, se refiere a la doxa bajtiniana que en occidente ignoró el contexto histórico y filosófico de índole espiritualista del autor ruso. Sostiene que Bajtín escribió acerca del acto del sujeto individual y la relación psicológica interindividual, mientras que Voloshinov se situó entre la sociología y la psicología, en la búsqueda de una conceptualización materialista del lenguaje, en la relación social, entre lo colectivo y lo individual.

Como señalara Ivanova (2015), Voloshinov amplió la noción de diálogo y la extendió a cada producción verbal, lo que permitió que después Bajtín (1982/1999), hablara de la polifonía de la novela. Pero la complejidad del concepto de diálogo que introduce Voloshinov (2009) radica en la doble articulación de la conciencia a través del carácter semiológico de la interacción verbal. La entidad del signo ideológico es colectiva e individual a la vez, en la medida en que la conciencia individual participa de la colectiva por un proceso de reflexión y refracción.

Si los estudios y análisis de los textos de los años 20-30 han aportado acerca de la verdadera construcción histórica rusa del dialogismo y el malentendido francés, en lo que respecta al concepto de géneros discursivos, también ha sido aclarado, ya que el término discurso no existe en ruso, el concepto de géneros discursivos de occidente es otro, no surgió del contexto ruso, sino del contexto histórico-cultural de los años 60 de Francia. Si bien en inglés géneros discursivos fue traducido como "géneros de habla", tratándose de una traducción más próxima al sentido en ruso y con mayor precisión, Sériot $(2008 ; 2010)$ introduce y propone el término "géneros de la palabra" o "géneros del habla o del lenguaje".

En su investigación sobre el macrocontexto de los años 20-30 en la URSS, Tylkovski (2012) sostiene que es necesario revisar las influencias pasadas y presentes de los intelectuales que se sabían iniciadores de nuevas tradiciones, quienes estaban insertos en una revolución social que criticaba las construcciones intelectuales y científicas occidentales. Conocían la producción teórica europea y norteamericana que arribaba a Petrogrado/Leningrado a través de Francia y Alemania. Por eso esta autora encuentra diversas influencias en Voloshinov: 
"la concepción de "interacción verbal" (con las influencias rusas de interacción interindividual, recibidas de Sorokin) en relación con la de "diálogo" (con la influencia de Jakubinskij) la noción de la "naturaleza social del enunciado" (con la influencia sociológica de Roberty) conforman una construcción teórica compleja". (RIESTRA, 2015)

De nuestra parte, en los estudios lingüísticos, tanto de Jakubinskij como de Voloshinov, observamos un enfoque psicológico del lenguaje. Según Ivanova (2010) se trata de la influencia de Baudoin de Courtenay (1845-1929), quien fuera profesor de Jakubinskij. De él proviene la concepción de la funcionalidad del lenguaje, en el sentido desarrollado por Humboldt respecto de la diversidad funcional y de otorgar el mismo valor a todas las lenguas existentes. Para Ivanova (2010) el concepto de actividad de lenguaje que circuló entre lingüistas, filósofos y psicólogos de la época pos revolucionaria rusa, entre ellos, Jakubinskij, Voloshinov, Medvedev, Vygotski, Luria, Leontiev, etc. se sostiene en el enfoque psico-social del lenguaje y la lingüística que desarrollara Baudoin de Courtenay.

Hay, asimismo, otra influencia decisiva en los autores rusos de esta década, que es la de Williams James (1767-1835), el filósofo-psicólogo norteamericano que estudió el aspecto emocional del lenguaje y la explicación de la percepción como fenómeno. En particular, observó lo global y lo local como dimensiones del diálogo y éste como acción voluntaria simple, conceptos que tomará Jakubinskij directamente, citándolo a este autor en diversos trabajos. Las influencias ponen de manifiesto hasta qué punto los lingüistas y psicólogos rusos de la década 20-30 estaban contactados con la producción intelectual occidental, una situación que no se produjo a la inversa.

\section{El análisis del diálogo en la sala de aula}

En publicaciones anteriores (RIESTRA, 2014 y 2017) nos basamos en los conceptos de actividad de lenguaje y de diálogo de los rusos y, en particular, a partir del concepto de habla dialogal, desde donde estamos reformulando el enfoque metodológico de investigación de la interacción en las clases de Lengua y literatura de la escolaridad básica. 
En el análisis de Jakubinskij la diversidad funcional aparece en los diálogos reales en contraposición con monólogos (orales o escritos); el proceso de hablar es observado y definido como automatismo verbal (reacción natural espontánea de la réplica), por lo que el diálogo es el fenómeno cultural cercano a lo biológico (psicobiológico, dirá él), un fenómeno de la naturaleza.

Esta diferenciación entre diálogo y monólogo delimitó como natural el primero y artificial el segundo, a partir de los planteos de Scherba, quien fuera profesor de Jakubinskij. Éste profundiza el análisis al llevar el objeto de estudio al plano empírico y delimitar el fenómeno del diálogo como "habla dialogal" o"interacción":

Esencialmente, toda interação entre os individuos é necesariamente uma inter- ação. Em razão de sua natureza, ela busca evitar a unilateralidade, esforçando-se para ser bilateral, "dialógica" e foge do "monólogo". (JAKUBINSKIJ, 2015, p. 76)

Desde la perspectiva interaccionista sociodiscursiva en la que nos situamos, el aula como objeto de estudio es el espacio verbal situado que investigamos en el campo de Didáctica de las lenguas.

Las observaciones etnográficas como registros naturales nos permiten analizar las interacciones verbales entre los profesores y los alumnos, tanto de nivel primario, como de secundario. El objeto de estudio específico ha sido la consigna de instrucciones de tareas que los profesores dan a sus alumnos para presentar los objetos de enseñanza de cada clase y las representaciones de los objetos de enseñanza que nos permiten entender la transposición didáctica de las clases.

La teoría de Jakubinskij nos abrió una perspectiva situacional y, sobre todo, contextual para analizar el diálogo real en cada clase entre el profesor y sus alumnos. Nos basamos en las réplicas (término que Jakubinskij creara especialmente en ruso replicirovanie- ) para analizar: a) lo inacabado del diálogo (cada réplica supone una contra -réplica) y b) las interrupciones como necesarias en la interacción, ambas características señaladas entre otras, a partir de la observación empírica.

Por lo tanto, la unidad de análisis de la réplica nos permitió enfocar los enunciados de la clase bajo otra mirada que incluyó las reacciones de los alumnos a las palabras de la profesora o el profesor para comprender el espacio comunicativo en el aula. 
En la descripción del habla dialogal Jakubinskij introduce la noción de percepción. Es lo que permite indagar el proceso de la interiorización de la palabra del otro y el efecto que produce la réplica del otro.

De este modo, uno de los aspectos no verbales o complementarios de la verbalización dialogal es el de la percepción y la apercepción, en relación con la comprensión y la continuidad de la comunicación.

La comprensión por conjetura y el hecho de hablar por alusiones (cuando se sabe de qué se trata), tiene una cierta comunidad de masas aperceptivas de los interlocutores (en el sentido que James apuntara como conjunto de experiencias y saberes anteriores necesarios para comprender e interpretar un enunciado). El hablar por alusiones o la suposición tiene un papel considerable durante el intercambio verbal y es lo que hace posible el uso definido o indefinido de las palabras concretas. La importancia de las masas aperceptivas es apuntada por Jakubinskij (2015) como la condición y posibilidad de desarrollarse una lengua:

Em fim, a comunidade de massas aperceptivas em um dado meio condiciona um dos fatos fundamentais do desenvolvimento da lingua: a formaçao dos diferentes dialetos sociais com suas particularidades no léxico, no emprego das palabras, a sintaxe, etc. (JAKUBINSKIJ, 2015, p. 97)

Esta referencia al habla y su funcionalidad también fue objeto de análisis de Coseriu (1992) y podemos observar la proximidad epistemológica entre el concepto de lengua funcionalcoseriano y las variables del habla de Jakubinskij.

Para Coseriu la lengua funcional es coincidente con el concepto de dialecto y sin lenguas funcionales no habría lenguas históricas:

En cada punto del hablar sólo puede realizarse una determinada lengua funcional. Sin embargo, en el hablar se pueden realizar perfectamente varias lenguas funcionales, aunque en diferentes puntos de la misma habla o del mismo texto.(COSERIU, 1992, p. 39).

El análisis funcional de Jakubinskij no es el de la "funcionalidad" de Jakobson, sino la "diversidad funcional" humboldtiana, que distingue "diversidad de formas". La diversidad funcional fue observada en diálogos reales, es decir, prestó atención a la fonación antes de que la sociolingüística surgiera como enfoque disciplinar en los años 6o. La coincidencia epistemológica que encontramos con Coseriu, se debe a que ambos analizaron el uso de las lenguas y, en el caso de Jakubinskij la mención a lo 
extraverbal constituye un avance respecto de los enfoques formales al estilo de los neogramáticos, lo que tanto cuestionó Saussure, sin que lograra comprenderse en la época:

Entretanto, no nosso caso, partindo da distinção das formas da fala, estabelecemos uma ponte entre os fatores extralinguísticos e os fenômenos verbais, e temos, assim, a possibilidade de falar, por exemplo, da distinção dos meios de informação nessa ou naquela variante, ou de opor diretamente monólogo e diálogo como fenômenos verbais." (JAKUBINSKIJ, 2015, p. 61)

De estas relaciones conceptuales coincidentes entre ambos autores extrajimos algunas categorías de análisis para nuestra investigación en la enseñanza de la lengua y la literatura que resumimos a continuación.

Tabla 1. Habla dialogal y consignas

\begin{tabular}{|l|l|l|}
\hline $\begin{array}{l}\text { Habla dialogal } \\
\text { automática }\end{array}$ & $\begin{array}{l}\text { Espacio verbal/mental } \\
\text { consignas }\end{array}$ & $\begin{array}{l}\text { Masas aperceptivas } \\
\text { Alumnos y profesores }\end{array}$ \\
\hline $\begin{array}{l}\text { Se entiende según los } \\
\text { supuestos }\end{array}$ & Decir para que hagan & $\begin{array}{l}\text { No coincidencia } \\
\text { naturalmente por lenguas } \\
\text { funcionales /dialectos } \\
\text { diferentes }\end{array}$ \\
\hline $\begin{array}{l}\text { Las nociones técnicas vs el } \\
\text { sentido común }\end{array}$ & $\begin{array}{l}\text { Situarse en la mente del } \\
\text { otro }\end{array}$ & $\begin{array}{l}\text { Espacio a construir de } \\
\text { diálogo en colaboración: } \\
\text { alusiones comunes }\end{array}$ \\
\hline
\end{tabular}

En la sala de aula el diálogo es lo que realmente sucede lenguajeramente entre docente y alumnos, es lo incontrolado, automático, naturalizado de las lenguas funcionales diferentes entre quien enseña y quienes aprenden.

La toma de conciencia de esta diversidad lingüística funcional nos condujo a revisar la metodología para conjugar el análisis de la consigna con el análisis de las masas aperceptivas de la clase de Lengua, como mostramos en la Tabla 1.

Lo planificado (consignas) y lo natural no planificado (diálogo) necesitan integrarse como dos órdenes comunicativos diferenciados. Prestar atención al diálogo, pese a no estar incorporado su estudio en la formación profesional actual, podría ser una dimensión novedosa de análisis del trabajo docente desde esta perspectiva dialógica. La perspectiva dialógica que proponemos se organiza desde las 
lenguas funcionales y las masas aperceptivas. La coincidencia de masas aperceptivas entre docente y alumnos sería una consecuencia deseable de la interacción. Como las masas aperceptivas son las que condicionan las lenguas funcionales o dialectos sociales, este intercambio verbal de la sala de aula que en la actualidad presenta dificultades comunicativas, al ser analizado por los mismos profesores, a través de los registros etnográficos, podría mostrar posibles encuentros discursivo-textuales, necesarios para la comunicación y el conocimiento escolar.

El análisis del diálogo tendría la utilidad de conformar un espacio de articulación didáctica para auto-observar las clases.

Los supuestos de las masas aperceptivas pueden analizarse a través de las lenguas funcionales diferentes y esta información podría contribuir en la construcción, desde el rol docente, de un diálogo colaborativo, en la medida en que pueda analizarse y revisarse el diálogo real de la clase.

\section{El diálogo en Voloshinov a partir del enunciado como fundamento del concepto de género}

Seis años después de la definición del objeto de estudio de Jakubinskij respecto de las distinciones funcionales de Humboldt entre lenguaje hablado, lenguaje poético, lógico-científico, lenguaje del orador, el concepto de "género de la palabra" fue delimitado y desarrollado en Marxismo y filosofía del lenguaje (1929) por Valentin Voloshinov quien, de acuerdo con Ivanova (2012) y Tylkovski (2012), estuvo en contacto directo con Jakubinskij entre los años 1923 y 1926.

Como señalamos, Voloshinov( 2009: 182 y 231) reconoce personalmente que el concepto de diálogo fue elaborado por Jakubinskij. Sin embargo, es necesario destacar la diferencia epistemológica o, más precisamente, la derivación conceptual aportada por Voloshinov respecto del habla dialogal, que consiste en la profundización del análisis del carácter extraverbal de la conformación del diálogo, a partir de la observación formal de las situaciones concretas, es decir, la producción social del enunciado:

Todo enunciado, por más terminado e importante que fuese en sí mismo, es tan sólo un momento en la comunicación discursiva continua (cotidiana, literaria, cognoscitiva, política). Pero además, este intercambio discursivo es, a su vez, tan sólo un momento de un 
continuo y multilateral proceso generativo de un colectivo social determinado. De ahí surge un problema importante: el estudio del vínculo entre una interacción concreta y una situación extraverbal más próxima $y$, a través de ésta, la relación con la situación más amplia. (VOLOSHINOV, 2009, p. 153)

Los estudios de Voloshinov se basan en la incipiente sociología y la determinación de lo social, de allí que enfocara lo genérico, abriendo una concepción nueva en el estudio del lenguaje humano.

Jakubinskij analizó diálogos literarios y, en algunos casos, utilizó las referencias concretas de discusiones académicas; no obstante, al finalizar su artículo sobre el habla dialogal aconseja la tarea colectiva frente a este nuevo objeto de estudio que se propuso delimitar. Sostuvo que debía realizarse un gran número de grabaciones de diálogos reales. Podemos deducir que fue Voloshinov quien tomó el mensaje y, más allá del estudio estrictamente lingüístico, extendió el campo hacia los estudios filosóficos y sociológicos del lenguaje, introduciendo un nuevo concepto de diálogo.

Por uma parte, Jakubinskij observó la comprensión, junto a la percepción y la apercepción (manifestadas en la entonación, en lo inacabado) para delimitar el concepto de habla dialogal, mientras, por outra parte, fue Voloshinov quien se situó frente a la producción del enunciado del otro para definir el diálogo, avanzando sobre el análisis de lo extraverbal como determinante, que señalara Jakubinskij; en esta dirección, Voloshinov (2009:145) formula la "ideología cotidiana" y analiza la interacción y la interiorización de la palabra del otro, así como el papel del signo ideológico en la doble articulación de la conciencia colectiva e individual.

La introducción del concepto de género por Voloshinov es otra novedad epistemológica porque pone de manifiesto cómo lo extraverbal determina la actividad lenguajera o verbal; de esta manera fundamenta el concepto de género de la palabra o género de la vida cotidiana, que es producto de las actividades no verbales, que se producen en contextos situacionales diferentes, lo que ejemplifica como "actos de trabajo, actos simbólicos de rituales", etc.

Según Ivanova (2010):

Al desarrollar sus reflexiones sobre la interacción social y su papel en la producción de un enunciado, sobre la influencia del auditorio en la forma del enunciado, Voloshinov llega a la idea de los géneros verbales que unas veces denomina "géneros de la vida cotidiana" (zitejskiezanry) y otras "géneros verbales de la vida" 
(ziznnenyerecevyezanry). En todos los casos la noción base es "género de la vida cotidiana" (ustojcivajabytovajasituacija) (IVANOVA, 2010, p. 58)

Para entender el concepto de género de la palabra, del lenguaje o de la vida cotidiana de Voloshinov, es necesario considerar el fondo ideológico-teórico del marxismo en la época, que tuvo, sin dudas, una influencia relevante. Pero más allá de la influencia del aire del tiempo, hay una tradición cultural rusa que atraviesa la concepción de actividad de lenguaje que puede observarse en autores como Jakubinskij, Medvedev, Vygotski, Luria, Leontiev y Voloshinov. La concepción rusa del lenguaje heredada tiene raíces profundas en la perspectiva de la percepción de los fenómenos empíricos y su análisis del lenguaje en este plano, como empirias, no como ideas acerca de los datos empíricos elegidos. Como sostiene Sériot (2008): "Se prestó atención a grandes nombres, pero se ignoró la riqueza considerable de Rusia como laboratorio de las ciencias humanas y sociales".

Traducido esto en términos pedagógicos, el interés radica en cómo introducir la noción de género de la palabra en el aula como objeto de enseñanza de las lenguas. Asimismo nos preguntamos qué debemos enseñar de los géneros para que esos conocimientos tengan el efecto psicológico de apropiación, interiorización y utilización eficaz en el proceso de desarrollo comunicativo verbal o lenguajero de cada alumno.

En la actualidad, las descripciones técnicas, tanto de la lingüística cognitiva como de la lingüística textual, denominadas tipos de textos o géneros de discurso no han tenido eficacia en el desarrollo del nivel praxiológico de la lengua en uso. Funcionan como simples descriptores de un objetivismo abstracto, que no produce efectos en los estudiantes en lo que hace a procesos cognitivos ni a desarrollos estructurales de los conocimientos lingüísticos adquiridos en la escuela.

\section{1 Los géneros de la palabra como géneros textuales escolares}

Retomemos el concepto de género de Voloshinov frente a la complejidad del objeto de estudio de la filosofía del lenguaje desde un enfoque sociológico, incluyendo el signo lingüístico en tanto signo ideológico que se produce en enunciados vinculados a interacciones comunicativas concretas: 
El lenguaje vive y se genera históricamente en la comunicación discursiva (verbal) concreta y no en un sistema lingüístico abstracto de formas, ni tampoco en la psique individual de los hablantes. Por consiguiente, un orden metodológicamente fundado del estudio del lenguaje debe ser el siguiente:"1) formas y tipos de interacción discursiva en relación con sus condiciones concretas; 2) formas de enunciados concretos, de algunas actuaciones discursivas en estrecha relación con la interacción cuyos elementos son estos enunciados, esto es, los géneros de las actuaciones discursivas, determinados por la interacción discursiva, en la vida y en la creación ideológica; 3) a partir de ahí, una revisión de las formas del lenguaje tomadas en su versión lingüística habitual. El mismo orden vale para una generación concreta del lenguaje: primero se genera la comunicación social (fundada sobre las infraestructuras), en ella se generan la comunicación y interacción discursiva y, finalmente, esta interacción se refleja en el cambio de las formas de la lengua. (VOLOSHINOV, 2009, p. 153-154).

Si el género es lo que permite ligar el contexto y la situación de la comunicación, aprendemos los géneros a lo largo de nuestras vidas en la medida en que los usamos, por lo tanto, el género es un principio organizador de los enunciados como productos del lenguaje. Se desprende de aquí que la situación comunicativa o contexto de producción de todo enunciado tiene una composición genérica.

La incorporación del concepto de texto que propusiera Bronckart (1997) como sinónimo de enunciado en el sentido utilizado por Volsoshinov, es un aporte a la didáctica de las lenguas que sintetiza la noción de texto como producto empírico, a la vez que proceso de producción verbal.

En este marco teórico del interaccionismo sociodiscursivo, el género es un instrumento comunicativo determinado por y producido en la práctica. Es un saber hacer para poder decir lo que esperan que digamos en determinadas situaciones sociales. Es un saber hacer normado, que adoptamos, pero, a la vez, adaptamos en cada situación comunicativa concreta, según lo plantea Bronckart (2007). De esta adaptación en cada uso surge la relativa estabilidad y el cambio permanente de los géneros, algo semejante a lo que ocurre con los signos lingüísticos en la comunicación humana, según los aportes de Saussure (2004).

En esta perspectiva teórica de índole sociológica e interaccionista de la creación sígnica, los géneros de textos o géneros textuales nos permiten repensar su transposición didáctica en los distintos niveles del sistema educativo. 
Los conceptos de signo y texto transpuestos como objetos de enseñanza se relacionan con el concepto de género de la palabra de Voloshinov, a partir de los aportes didácticos de Bronckart y Schneuwly (1991).

De aquí surge la propuesta de estos autores de organizar las secuencias didácticas en módulos o talleres, a partir de los géneros textuales, de acuerdo con las capacidades discursivo-textuales ya desarrolladas por los alumnos en relación con las capacidades a desarrollar aún.

En este marco de la Didáctica de las lenguas Schneuwly (1994) desarrolló el concepto de género como megaherramienta por ser un objeto de enseñanza escolar que incluye conocimientos lingüísticos como otras herramientas subsumidas en cada género, desde el sistema ortográfico, en la escritura, el sistema verbal, los conocimientos morfológicos para la construcción sintáctica en cada lengua, etc.

Por otra parte, para que los géneros se transformen en herramientas (a ser usadas como formatos comunicativos relativamente estables en la escuela) es necesario emprender la tarea investigativa de modelización del género elegido, el que será presentado en concreto a los alumnos con textos singulares, portadores de diversos aspectos del "hacer textual" en ese género.

La organización textual y las propiedades genéricas serán estudiadas por el profesor y seleccionadas de acuerdo con el nivel educativo y las capacidades discursivo-textuales desarrolladas de los alumnos. En este sentido, las masas aperceptivas de profesor y alumnos necesitan aproximarse frente al objeto de enseñanza concreto, que es el texto correspondiente a determinado género textual en la relación conceptual que proponemos esquematizada en la tabla 2.

Tabla 2 Los géneros textuales en el diálogo de la clase

\begin{tabular}{|l|l|}
\hline Géneros textuales/ Voloshinov & Diálogo profesor-alumnos/Jakubinskij \\
\hline $\begin{array}{l}\text { Organización textual y propiedades } \\
\text { genéricas }\end{array}$ & $\begin{array}{l}\text { Masas aperceptivas diferentes en } \\
\text { lenguas funcionales }\end{array}$ \\
\hline Comprensión y producción de textos & Signos lingüísticos \\
\hline
\end{tabular}


En este esquema se pretende mostrar el objeto de enseñanza (textos en géneros) y, a la vez, cómo se transmite ese objeto de enseñanza. Se trata de un saber hacer práctico que tenemos que transmitir en el diálogo de la clase.

Se nos presenta un cambio epistemológico (Riestra, 2006, 2010) que focaliza lo que Voloshinov describe como: 1) formas y tipos de interacción discursiva; 2) formas de enunciados concretos; 3) formas del lenguaje tomadas en su versión lingüística habitual.

Son tres dimensiones que en el trayecto didáctico de uso-sentido-forma hemos didactizado de este modo: el cómo hacer (uso) para elaborar el texto (sentido) con determinadas características lingüísticas (forma). Destacamos aquí que la forma lingüística es el último componente sobre el que reflexionamos, puesto que la determinación contextual es lo primero que consideramos al textualizar (hablando o escribiendo)

Por otra parte, la elaboración de las consignas, como textos planificados antes de la clase para proponer las tareas a los alumnos, serán reformuladas como textos no planificados durante el diálogo de la clase, es decir, serán traducciones posibles de nuestra lengua funcional con su jerga técnico-lingüística.

De este modo,desde el espacio dialogal (masa aperceptiva de otro/s alumno/s) se pasa al espacio monologal, generalmente escrito de la tarea, que es artificial según Jakubinskij- y complejo. Esta cuestión también debería ser objeto de análisis didáctico.

En definitiva, enseñar textos en géneros y recortar objetos de enseñanza a través de consignas es la tarea docente que se modifica necesariamente en el diálogo de la clase.

\section{Conclusiones}

Los esbozos metodológicos señalados constituyen una propuesta para discutir problemáticas actuales de interacción y enseñanza de las lenguas.

La posición epistemológica de Jakubinskij, basada en algunos principios respecto del habla dialogal tendrá influencia en Voloshinov y en Vygotski, dos autores que aún nos presentan programas temáticos significativos y vigentes. 
En particular, continúa siendo de interés y necesidad estudiar el principio de la formación e interiorización del lenguaje externo, lo que comprende el concepto central de actividad de lenguaje o lenguajera. Continúa siendo nuestro objeto de investigación didáctica lo que Jakubinskij colocara en 1923:

Como no início, durante o periodo de aprendizagem, cada atividade linguageira é uma atividade complexa e inhabitual. A atividade linguageira automática pertence ao tipo de atividades automáticas secundarias, que se formaram a partir das atividades conscientes por meio de repetiçao, exercício e hábito. (JAKUBINSKIJ, 2015, p. 110).

Podemos decir que la actividad del lenguaje descripta con precisión en las producciones teóricas de Vygotski, Luria y Leontiev, tanto respecto de la ontogénesis como de la filogénesis del desarrollo humano, se inscribe en estos principios del diálogo en la percepción del habla del otro.

En definitiva, la filosofía del entimema de Volsoshinov continúa siendo un principio orientador para el diálogo con los alumnos entre quienes enseñamos la asignatura escolar Lengua y literatura.

\section{Referencias bibliográficas}

BAJTIN, M. Estética de la creación verbal. México: Siglo XXI, 1982/ 1999.

BERTAU, M.C. Pour une notion de forme linguistique comme forme vécue. Une approche avec Jakubinskij, Volosinov et Vygotski. In: Langage et pensée: Union sovietique années 1920-1930. Lausanne: UNIL, 2008.

BRONCKART, J.-P. Activité langagière, textes et discours. Pour un interactionisme sociodiscursif. Paris: Delachaux et Niestlé, 1997.

Desarrollo del lenguaje y didáctica de las lenguas. Buenos Aires: Miño \& Dávila, 2007.

BRONCKART, J.-P; SCHNEUWLY, B. La didáctica de la lengua materna: el nacimiento de una utopía indispensable", Textos de Didáctica de la Lengua y de la Literatura, N9, julio, 1991.

BRONCKART, J.-P.; BOTA, C. Bakthine démasqué. Historie d'un menteur, d'une escroquerie et d'un délire collectif. Genève: Droz, 2011.

BUBNOVA, T. Prólogo y traducción de Marxismo y filosofía del lenguaje. Buenos Aires: Godot, 2009. 
COSERIU, E. Competencia lingüística. Madrid: Gredos, 1992.

IVANOVA, I. Les sources de la conception du dialogue chez $L$. Jakubinskij. Texto! diciembre de 2003 [en línea]. Disponible en:

$<$ http://www.revue-texto.net/Inedits/Ivanova_Jakubinskij.html> Acesso em 04 de junho de 2018.

El diálogo en la lingüística soviética de los años 1920-1930 en Riestra (ed.) Saussure, Voloshino y Bajtin revisitados. Buenos Aires: Miño \& Dávila, 2010.

Lev Jakubinskij, une linguistique de la parole. Lausanne: Lambert Lucas, 2012.

JAKUBINSKIJ, L. Sobre a fala dialogal. Sériot e Ivanova, eds. São Paulo: Parábola Editorial, 2015.

JAMES, W. Principios de psicología. México: Fondo de Cultura Económica, 1994.

LEONTIEV, A. El desarrollo del psiquismo. Madrid: Akal, 1983.

RIESTRA, D. Usos y formas de la lengua escrita. Buenos Aires:Noveduc, 2006.

Saussure, Voloshinov y Bajtin revisitados. Estudios históricos y epistemológicos. Buenos Aires: Miño\& Dávila, 2010.

Saussure, Vygotski, and Voloshinov. The linguistic sign as an epistemological issue. Language and Language Behavior, the Journal of the Linguistic Society of St. Petersburg, vol 14, 2014.

El contexto y la obra de Voloshinov-las controversias ideológicas- casi un siglo después. Saga Revista de Letras $\mathrm{N}^{\circ} 3$. Primer Semestre, 2015.

La concepción del diálogo de los rusos desde la perspectiva interaccionista socio-discursiva, Veredas - Interacionismo Sociodiscursivo, 2017/1, PPG Línguística/UFJF - Juiz de Fora, p. 11 - 29, 2017.

SCHNEUWLY, B. Genres et types de discours: considérations psychologiques et ontogénétiques, en Les interactions lecture-écriture. Actes du colloque Théodile -Crel (Lille, nov. 1993). Bern: Peter Lang, 1994.

SÉRIOT, P. Generalizar lo único: géneros, tipos y esferas en Bajtin. In: Riestra, D. Saussure, Voloshinov y Bajtin revisitados. Estudios históricos y epistemológicos. Buenos Aires: Miño \& Dávila, 2010.

Russie, linguistique et philosophie. Cahiers de I'ILSL, Lausanne, 2011.

Volosinov e a filosofía da linguagem. Sao Paulo: Parábola, 2015. 
SÉRIOT, P.; FRIEDRICH, J. Langage et pensée: union Sovietique années 1920-1930. Cahiers de l'ISLn 24, Lausanne, 2008.

TYLKOWSKI, I. Voloshinov en contexte. Éssaid'épistemologiehistorique. Limoges: Lambert- Lucas, 2012.

VOLOSHINOV, V. Marxismo y filosofía del lenguaje. Buenos Aires: Godot, 1929/2009.

VYGOTSKI, L. Pensamiento y habla. Buenos Aires: Colihue, 2009.

\footnotetext{
' Dora Riestra, Profesora en Letras (Universidad Católica de Santa Fe, Argentina)- Doctora en Ciencias de la Educación (UNIGE, Suisse).

Fue Profesora Titular Ordinaria de Didáctica de la Lengua y la Literaturahasta 2016, actualmente es Profesora Consulta de la Universidad Nacional de Río Negro, Argentina.

dora.riestra@gmail.com

driestra@unrn.edu.ar
} 